VOL. 52 (1995) [527-528]

\title{
Some aspects of homogeneous linear recursive sequences of order mainly two
}

\author{
Raymond Samuel Melham
}

This thesis contains results concerning aspects of homogeneous linear recursive sequences of order mainly two. Since the work focuses on the original research of the author, known results are recorded only to assist in the development or to demonstrate their relationship with the results of the author.

The first chapter states the objectives of the thesis and introduces the types of sequences under consideration. These include pairs of fundamental and primordial sequences. All second order sequences considered are special cases of Horadam's sequence $\left\{W_{n}\right\} \equiv\left\{W_{n}(a, b ; p, q)\right\}$ defined by

$$
W_{n}=p W_{n-1}-q W_{n-2}, \quad W_{0}=a, \quad W_{1}=b, \quad n \geqslant 2 .
$$

The second chapter is concerned with obtaining generalizations of identities involving simple products. Several of these generalizations are used subsequently in the thesis. The chapter concludes with a generalization of the Catalan identity.

The theme of the third chapter is summation. Both finite and infinite sums are considered, and they include results involving inverse trigonometric and inverse hyperbolic sums. The sequences under consideration are the Pell related and Chebyshev related sequences defined in the first chapter.

Reciprocal summation is the theme of the fourth chapter. The well known method of telescoping sums is used frequently. Results for Chebyshev related sequences which parallel existing results for Pell related sequences are established. Finally, a technique is extended by which third order sequences are used to derive reciprocal sums for second order sequences.

Various results involving congruence and divisibility occur in Chapter five. A large proportion of the results of this chapter involve congruences with prime power moduli. For example, a generalization of the following known result is given:

Received 25th May, 1995

Thesis submitted to University of Technology Sydney, December 1994. Degree approved May 1995. Supervisor: Professor A.G. Shannon.

Copyright Clearance Centre, Inc. Serial-fee code: 0004-9729/95 \$A2.00+0.00. 
Let $P$ be prime. If $\Delta=p^{2}-4 q$ then

$$
\begin{aligned}
V_{k P^{n}} & \equiv V_{k P^{n-1}}\left(\bmod P^{n}\right), \quad \text { all } P, \\
U_{k P^{n}} & \equiv\left(\frac{\Delta}{P}\right) U_{k P^{n-1}}\left(\bmod P^{n}\right), \quad \text { for } P \text { odd and } P \nmid \Delta, \\
U_{k 2^{n}} & \equiv(-1)^{q} U_{k 2^{n-1}}\left(\bmod 2^{n}\right),
\end{aligned}
$$

where $\left(\frac{\Delta}{P}\right)$ is the Legendre symbol, and $\left\{U_{n}\right\}$ and $\left\{V_{n}\right\}$ are the fundamental and primordial sequences of Lucas respectively.

Many new infinite sums incorporating the trigonometric functions are derived in Chapter six. Matrices, used in conjunction with power series, play a vital role in the development of most of the results.

In Chapter seven, numerous finite sums are established using matrix methods. Most results depend upon the use of several 2-by-2 matrices. One such matrix is

$$
M_{k, m}=\left(\begin{array}{cc}
U_{k+m} & -q^{m} U_{k} \\
U_{k} & -q^{m} U_{k-m}
\end{array}\right)
$$

where $U_{n}=W_{n}(0,1: p, q)$. Furthermore, as an extension of work done using a 3by-3 matrix, a conjecture is made concerning the characteristic equation of an $n$-by- $n$ matrix, $S_{n}$, whose $(i, j)$ element is

$$
(-1)^{n-i}\left(\begin{array}{c}
j-1 \\
j+i-n-1
\end{array}\right) p^{i+j-n-1} q^{n-i}
$$

Results for third and higher order recurrences occur in Chapter eight. Some of the achievements in this chapter include the statement and proof of a combinatorial summation identity for polygonal numbers, a generalization of a result of D'Ocagne and an answer to an old question of Jarden. The chapter concludes with some conjectures which could lead to further research.

Chapter nine, the final chapter, contains a review and some ideas for further research.

3 Dickenson St

Panania NSW 2213

Australia 